\title{
Self Soothing by Reviewing Favorite Memories: An Exploration of Mobile Application Prototypes, Which Facilitate Positive Wellbeing via Reminiscing
}

\author{
Alice Good, Claire Ancient, Georgiana Postolache, Alexandru Socianu, Adam \\ Afghan \\ University of Portsmouth, PO1 3AE UK \\ alice.good@port.ac.uk; claire.ancient@port.ac.uk
}

\begin{abstract}
The premise of this paper is to promote the results from a small study which sought to evaluate the effectiveness of three different prototype mobile applications, in promoting well being by the reminiscing of positive memories. Interviews were carried out with mental health professionals, including psychotherapists and psychiatric nurses, who were questioned on their opinions as to the effectiveness of the proposed app after using the prototype. The results indicate a significant interest in the proposed tool, with all professionals recognizing the effectiveness of positive reminiscing and indicating a willingness to take part in subsequent prototype evaluation developments.
\end{abstract}

Keywords: Wellbeing, Mobile Applications, Reminiscing

\section{Introduction}

There is potential for using mobile applications as a means to facilitate the process of reminiscing, and this is the focus of the presented research. Whilst there are now a vast range of apps to support physical health, supporting mental health is still a new area, particularly in the area of behavioral therapy.

Mobile applications could be used to facilitate positive reminiscing, and as such, be utilized as a self soothing or distraction tool, for people experiencing low moods or anxiety (Good et al, 2012). The theory is based upon Reminiscent Therapy (RT), designed for people with dementia and involves using meaningful prompts, including photos, music and recordings as an aid to remembering life events (Norris, 1986). Some research states that it has been useful in reducing depression (Scogin F \& McElreath, 1994) as well as being an important tool to facilitate socialization. Positive reminiscing can also promote a sense of wellbeing.

The process of reminiscing as a self soothing tool is relatively under explored area. Whilst it has been predominantly utilized in people with dementia, there could be scope for applying the theory of RT in other mental health conditions, particularly where depression and general low mood are common Good et al, 2013). This could potentially induce a 'self soothing' process which could lend itself well to people who struggle with day to day living as a result of low mood, or indeed who experience the occasional 'off-day'. The act of 'self soothing', that is calming us down, is in fact one of the most difficult things to achieve when you have mental health problems. Yet 
the capability to be able to calm oneself down, to essentially self soothe, would be hugely advantageous to people with mental health problems, and could potentially prevent problems from escalating further, if only by means of a distraction. It is the premise of this research to develop apps that can help facilitate positive reminiscing.

\section{Mobile App Prototypes for Reminiscing}

This research presents examples of reminiscent applications. Three alternative prototype mobile applications were designed on IOS and Android platforms, to display meaningful memorabilia which could be used to promote positive wellbeing.

\subsection{Remember When}

'I Remember When' (Ancient et al, 2013) is designed as a tablet application, originally intended for people with dementia, but with potential to be utilised by wider user groups (See Figure 1). Users have the option of reminiscing with personal memories or memories through different eras. The latter option would be particularly suitable for people with dementia, for promoting discussion (Norris, 1984).
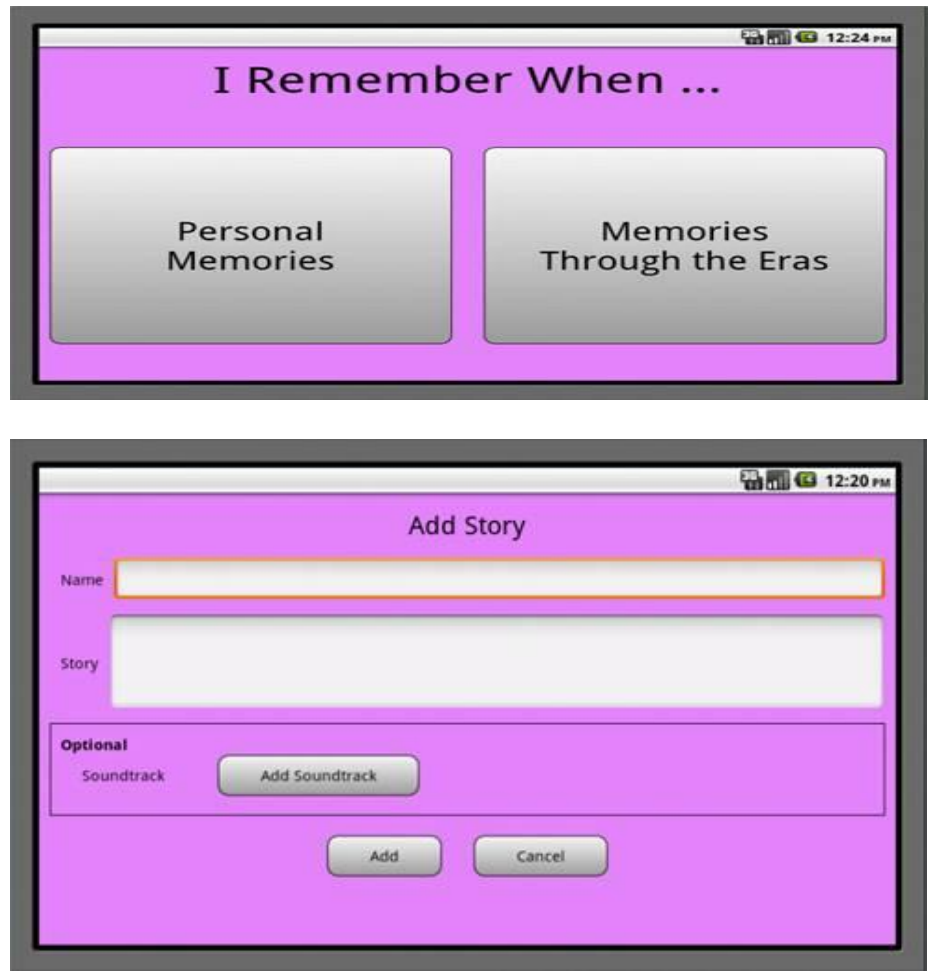

Fig. 1. Mobile App: I remember when 
The app also provides the facility for users to add personal stories about their lives, which could be related to certain events that triggered positive memories. There is also the facility to assign a relevant sound track to this story, which could enhance the reminiscence process.

\subsection{Remember Me}

The second prototype - 'Remember Me' was designed for IOS phones. Users can upload their favourite pictures, videos, audio and notes into the app. There is the facility to assign meaningful comments/notes to the memorabilia. In addition, users could copy and paste content from texts, emails and other files into the 'notes' section, for example poems or special messages (see figure 2)

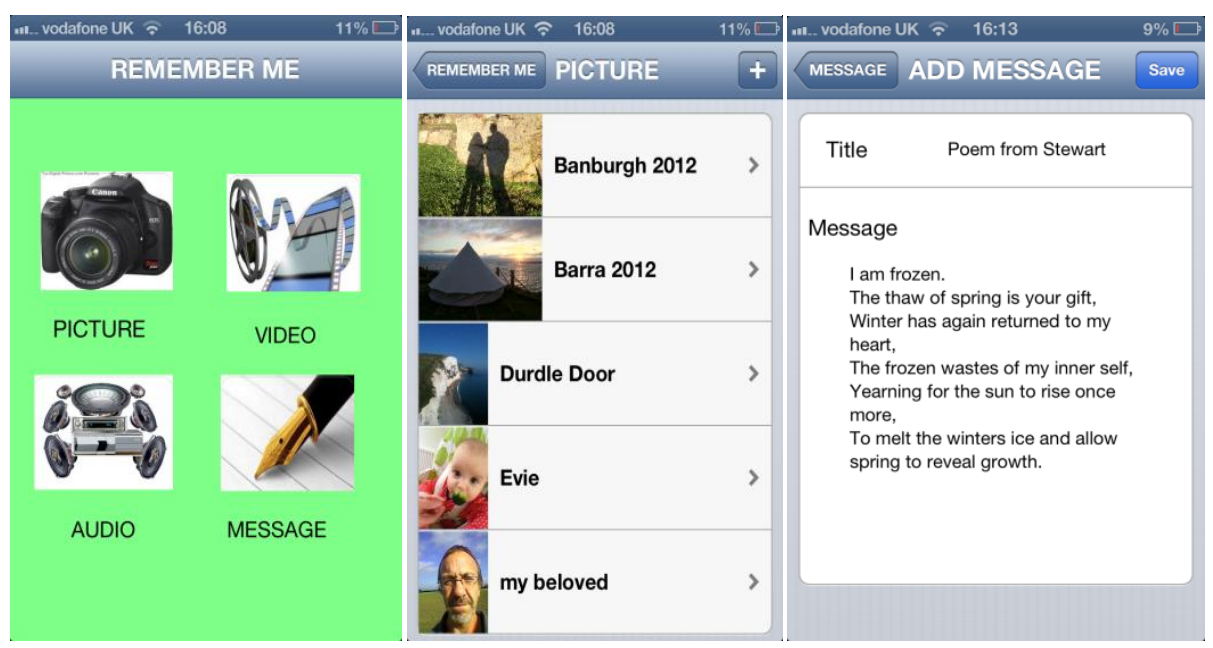

Fig. 2. Mobile App: Remember Me

\subsection{My Favourite Memories}

The final prototype, entitled 'My Favourite Memories' (see figure 3), was also designed as a tablet application but can also be used on Android phones. With this app, users can store favorite photos and music as well as meaningful notes. As with the previous apps, users can add descriptions or positive notes in the form of prompts to the uploaded media. 


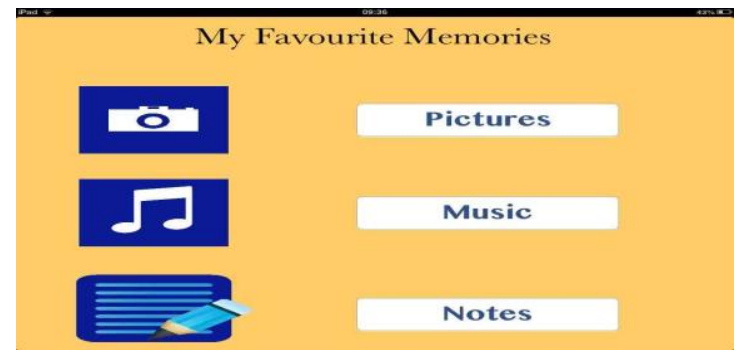

Fig. 3. Mobile App: My Favourite Memories

\section{Evaluation Study}

\subsection{Method}

In adopting a user-centered approach to design, the researchers carried out an evaluation of the prototype applications with six experienced professionals who support people with a range of psychiatric disorders, including anxiety, depression and personality disorder. These professionals, who were interviewed separately, were presented with prototypes of the three apps. They were then questioned on the following criteria: whether they thought the app would be suitable in promoting self soothing; likelihood of their clients using these types of apps; likelihood of clients becoming stressed using the app and lastly, whether they would be interested in participating in subsequent research using the proposed apps.

\subsection{Results}

Qualitative data was gathered and presented in table 1 below. Rigorous analysis using methods such as content analysis and categorization was not deemed appropriate given the very small sample group. This research should effectively be viewed as a pilot study and as such, the results viewed as indicative only.

Table 1. Professionals' Perspectives on the Effectiveness of Reminiscent Apps

\begin{tabular}{|l|l|}
\hline $\begin{array}{l}\text { Potential to aid self } \\
\text { soothing }\end{array}$ & $\begin{array}{l}\text { All professionals agreed that the ability to self soothe is very } \\
\text { important. General consensus was that these types of reminiscent } \\
\text { apps could potentially be used as a self soothing tool. }\end{array}$ \\
\hline $\begin{array}{l}\text { Likelihood of clients } \\
\text { using app }\end{array}$ & All clients had a mobile phone but not all used smart phones. \\
\hline $\begin{array}{l}\text { Likelihood of clients } \\
\text { becoming stressed } \\
\text { whilst using app }\end{array}$ & $\begin{array}{l}\text { Some issues with regards to getting started and maintaining the usage } \\
\text { of the app. }\end{array}$ \\
\hline $\begin{array}{l}\text { Interest in } \\
\text { involvement in } \\
\text { subsequent research }\end{array}$ & $\begin{array}{l}\text { Five out of six indicated an interest in becoming involved in any } \\
\text { subsequent research and trials using the app. }\end{array}$ \\
\hline
\end{tabular}




\section{Discussion}

Self soothing is one of the most difficult and challenging things to achieve when feeling stressed. All of the professionals questioned, agreed it is an important skill and can reduce the likelihood of behavioral problems and improve mental health, as well as potentially reducing the likelihood of clients requiring intervention from mental health services. Some professionals reported that not all of their clients had smart phones, others were not sure. A possible solution for this could be to ensure the apps could be used via laptops or desktop computers. Two professionals voiced concern in relation to clients becoming stressed using the app, but this was more in relation to the likelihood of clients requiring encouragement to get started and to maintain usage. Whilst the application can be set up by individuals, it could also be set up with the support of service providers, for example community nurses/support workers. Five out of the six professionals indicated an interest in becoming involved in related research which involved user testing for effectiveness and usability.

The results of the study suggest that mobile applications could be an effective, as well as accessible method to facilitate positive emotional wellbeing, when used as a self soothing tool. Further research is required to address design issues, particularly in relation to usability and user experience. The apps will need to be designed in such a way that they not only promote self soothing, but also a positive user experience, as clients could potentially be stressed prior to usage and will require an intuitive, easy to use app. Furthermore, studies will be required with end users to evaluate effectiveness. This study is part of a larger research project which focuses on the development of mobile applications for promoting self soothing and wellbeing.

\section{References}

Ancient, C. \& Good, A. (2013) Can Ubiquitous Devices Utilising Reminiscence Therapy be Used to Promote Well-being in Dementia Patients? An Exploratory Study. In: Universal Access in Human-Computer Interaction, part of HCI International 2013, 21st - 25th July 2013, Las Vegas (In Press).

Good, Alice, Sambhantham, A. and Panjganj, Vahid (2013) Looking back at Facebook content and the positive impact upon wellbeing: Exploring reminiscing as a tool for self soothing. In: Online Communities and Social Computing, part of HCI International 2013, 21st - 25th July 2013, Las Vegas (In Press)

Good, A., Wilson, C., Ancient, C., Sambhanthan, A. (2012).A Proposal to Support Wellbeing in People with Borderline Personality Disorder: Applying Reminiscent Theory in a Mobile App. The ACM conference on Designing Interactive Systems

Norris AD. (1986) Reminiscence with Elderly People. London: Winslow, 1986.

Scogin F, McElreath L. (1994) Efficacy of psychosocial treatments for geriatric depression: a quantitative review. Journal of Consulting \& Clinical Psychology (1994) 62:69-74 
